\title{
Metabolic and inflammatory health in SARS-CoV-2 and the potential role for habitual exercise in reducing disease severity
}

\author{
Frank E. Marino ${ }^{1}\left[\right.$ (] Nicole T. Vargas ${ }^{2} \cdot$ Melissa Skein $^{1} \cdot$ Tegan Hartmann $^{1}$
}

Received: 19 August 2021 / Revised: 23 October 2021 / Accepted: 25 October 2021 / Published online: 1 November 2021

(c) The Author(s), under exclusive licence to Springer Nature Switzerland AG 2021

\begin{abstract}
Introduction The rapid emergence and spread of SARS-CoV-2 in late 2019 has infected millions of people worldwide with significant morbidity and mortality with various responses from health authorities to limit the spread of the virus. Although population-wide inoculation is preferred, currently, there is large variation and disparity in the acquisition, development, and deployment of vaccination programs in many countries. Even with availability of a vaccine, achieving herd immunity does not guarantee against reinfection from SARS-CoV-2. Emerging evidence indicates that vaccines do not eliminate infection but protect against severe disease and potential hospitalisation. Therefore, additional strategies which strengthen the immune system should be strongly considered to assist in reducing the overall health care burden and stem the rate of infection. There is now substantial evidence that SARS-CoV-2 disease severity and death are linked to existing comorbidities such as cardiovascular disease, obesity, and metabolic disorders.

Purpose In this review, we discuss the potential medium-to-long-term strategy of habitual exercise and its relationship to targeted comorbidities and underlying inflammation as a protective mechanism against SARS-CoV-2 disease severity. Conclusion We conclude that engagement in habitual physical activity and exercise could be a strategy to mitigate the development of comorbidities and improve the response of the immune system, potentially reducing the risk of symptoms and life-threatening complications if infected.
\end{abstract}

Keywords ACE2 $\cdot$ COVID-19 $\cdot$ Co-morbidities $\cdot$ Cytokine storm $\cdot$ Exercise $\cdot$ Inflammation

\begin{tabular}{|c|c|c|c|}
\hline \multicolumn{2}{|c|}{ Abbreviations } & CVD & Cardiovascular Disease \\
\hline ACE2 & Angiotensin-converting enzyme-2 & CRP & C-reactive protein \\
\hline ARDS & Acute Respiratory Distress Syndrome & GCSF & Granulocyte-colony stimulating factor \\
\hline \multirow[t]{2}{*}{ CD4 } & Glycoprotein serving as co-recep- & HTN & Hypertension \\
\hline & tor for T-cell receptor & IL & Interleukin \\
\hline \multirow[t]{2}{*}{ CD8 } & Transmembrane glycoprotein serving as & IFN & Interferon \\
\hline & co-receptor for T-cell receptor & IFN- $\gamma$ & Interferon type II \\
\hline COPD & Chronic Obstructive Pulmonary Disease & MAS & Macrophage Activation Syndrome \\
\hline COVID-19 & Clinical disease caused by SARS-CoV-2 & MERS & Middle East Respiratory Syndrome \\
\hline & infection & MCP-1 & Monocyte chemoattractant protein-1 \\
\hline & & MIP-1 $1 \alpha$ & Macrophage inflammatory protein-alpha \\
\hline & & NK & Natural killer cells \\
\hline \multicolumn{2}{|c|}{ Responsible Editor: John Di Battista. } & RBD & Receptor-binding domain \\
\hline \multirow{2}{*}{\multicolumn{2}{|c|}{$\begin{array}{l}\text { Frank E. Marino } \\
\text { fmarino@csu.edu.au }\end{array}$}} & SARS & Severe Acute Respiratory Syndrome \\
\hline & & SARS-CoV-2 & Severe Acute Respiratory \\
\hline \multirow{2}{*}{\multicolumn{2}{|c|}{$\begin{array}{l}\text { School of Allied Health, Exercise and Sport Sciences, } \\
\text { Charles Sturt University, Bathurst, NSW 2795, Australia }\end{array}$}} & T2DM & Type 2 diabetes mellitus \\
\hline & & TLR & Toll-like receptor \\
\hline \multirow{2}{*}{\multicolumn{2}{|c|}{$\begin{array}{l}\text { Thermal Ergonomics Laboratory, Discipline of Exercise } \\
\text { and Sport Science, School of Health Sciences, Faculty } \\
\text { of Medicine and Health, University of Sydney, Camperdon }\end{array}$}} & $\mathrm{TNF}-\alpha$ & Tumour necrosis factor-alpha \\
\hline & & URTI & Upper respiratory tract infection \\
\hline
\end{tabular}




\section{Introduction}

The rapid emergence and spread of SARS-CoV-2 in late 2019 has infected millions of people worldwide with significant morbidity and mortality [1]. The clinical disease caused by SARS-CoV-2 was named COVID-19 by the World Health Organisation (WHO) to distinguish it from the more general coronavirus [2]. At the time of writing, the number of confirmed cases worldwide is over 242 million with over 4.9 million deaths [3]. The worldwide pandemic has resulted in a range of responses from national health authorities to limit the spread of the virus including: restricting social interactions, enforcing social distancing, wearing of masks, night-time curfews, and more extreme measures such as closing domestic and international borders. The success of these measures is still debated, although some countries such as New Zealand and Australia have considerably reduced case numbers and maintained zero cases of community transmission for extended periods of time despite minor outbreaks occurring [4]. These measures have largely been mandated for two main reasons. First, infection from SARS-CoV-2 can range from being asymptomatic or having mild symptoms to acute respiratory stress syndrome and death with protection by individual immunity unpredictable and variable [5]. Second, without implementing the mitigation measures, the overall spread and associated death count would arguably be much higher with even greater demand being placed on frontline workers and resources, than has thus far been documented. Although population-wide inoculation is preferred, currently, there is large variation and disparity in the acquisition, development, and deployment of vaccination programs in many countries [3]. The current state of vaccination is that approximately 3 billion doses have been administered worldwide [3] which is still significantly short of achieving herd immunity. Therefore, attenuating community transmission remains critical and the possibility of disease elimination should still remain attractive while waiting for worldwide vaccination to be at a level which can result in herd immunity [6].

Although there is no question that attempts to suppress community transmission have led to significant economic burden, particularly in countries where mitigation was adopted over virus elimination [7], the development, and now the availability of a vaccine needs to overcome practical problems. The allocation of resources and infrastructure for the vaccination of a large population may limit attempts to achieve herd immunity by vaccination [8]. In addition to these obstacles, there are significant sociocultural hurdles to overcome. For example, there is a large percentage of any population refusing to be vaccinated and others being hesitant [9]. This might be due to uncoordinated public-health messaging related to the type of vaccine that should be used for different age groups [10], or temporary recalls of some vaccines due to potential adverse events [11]. For example, in Australia, the restricted choice for the type of vaccine and the limited supplies of vaccines as a whole has stifled vaccination programs and extended the time to achieve potential herd immunity. In fact, at the time of writing, Australia had achieved zero-community transmission, but quarantine breaches resulted in transmission of the Delta variant of SARS-CoV-2 which is thought to be more transmissible and potentially lead to more severe symptoms [12]. This new variant and the potential for greater transmissibility forced local government authorities to mandate emergency restrictions such as lockdown for specific geographical locations.

Even with the availability of a vaccine, there are concerns that elimination of SARS-CoV-2 by vaccination and achieving herd immunity do not guarantee against reinfection from SARS-CoV-2, or a subsequent zoonotic disease which might erupt in a similar fashion as SARS-CoV-2. Emerging evidence indicates that vaccines do not eliminate infection, but only protect against severe disease and potential hospitalisation, so there will always be a portion of the population at risk - those that refuse to opt for vaccination even if there is herd immunity [13]. In addition, there is some uncertainty as to the duration of protection once vaccinated. Therefore, while vaccines are essential, additional strategies which strengthen the immune system should be part of the discussion as this can help to reduce the overall health care burden and stem the rate of infection, independent of additional measures that health authorities might employ.

One medium-to-long-term strategy is related to the response of the immune system to exercise. There is now good evidence, indicating that habitual, moderate exercise could in fact assist in risk reduction from upper respiratory tract infections (URTI) compared with a sedentary lifestyle [14]. Although the mechanism for improved immunity from habitual exercise is not fully understood, it appears that moderate training, especially in later life, is associated with maintenance of certain cellular immune function, such as $\mathrm{T}$ cells, and innate immunity [15]. A key outcome from engaging in habitual exercise is that it can ameliorate and even reduce the prevalence of chronic disease conditions such as cardiovascular disease and metabolic syndrome because of the attenuated chronic inflammation that is characteristic of these conditions [16]. In contrast, and as we now know, when individuals that have these and other comorbidities become infected with SARS-CoV-2, they are likely to experience more severe symptoms and potentially poorer outcomes [17]. Nevertheless, it remains unknown if there is a protective effect of habitual exercise against the severity of SARS-CoV-2 due to improved immune function despite 
some early evidence that greater aerobic capacity confers protection against severe disease and hospitalisation [18].

The broad aim of this review is to provide the reader with a brief history of the coronavirus to contextualise the current disease, to marshal the emerging evidence relating to SARS$\mathrm{CoV}-2$ morbidity and mortality relative to the pathophysiology, and finally, to highlight the importance of habitual exercise as a strategy to reduce the severity of SARS-CoV-2. If the theoretical proposition for an anti-inflammatory mechanism is viable, it may lead to pre-emptive intervention by habitual physical exercise as a protective measure against SARS-CoV-2 and potentially for other similar diseases. A secondary aim is to provide a contextual viewpoint which could assist professionals working in areas of allied health to further understand the pathophysiology of this virus and make educated decisions about implementing prescription of physical exercise as a key aspect of healthcare as a prevention strategy. At present, the relationship between SARS$\mathrm{CoV}-2$ and exercise is not at the forefront of mitigating the pandemic but could become important in the medium-tolong-term management of the disease.

\section{Origins, physiology, and symptomatology of coronaviruses}

According to the International Committee on Taxonomy of Viruses, SARS-CoV-2 belongs to the group of viruses generally labelled coronaviridae, which to date amount to 45 different species [19]. Coronaviruses were first described in 1965 by a group of virologists studying the common cold [20]. These researchers obtained a nasal swab from a child that presented with apparent common cold. Following a series of washings and testing, the inoculation for known viruses which they predicted would reveal influenza and other viruses such as rhinoviruses, they were unable to confirm the existence of such known viruses. However, they did confirm that their sample was indeed a "virus virtually unrelated to any other known virus of the human respiratory tract" (p. 1470). Imaging studies by Almeida and Tyrrell [21] revealed the distinct appearance on the virus particle surface covered with a distinct layer of projections which have a narrow stalk, giving it the resemblance of the solar corona [22].

Of the number of coronaviruses identified, three have caused serious worldwide population health concerns. In November 2002, a novel coronavirus, SARS emerged in China's Guangdong Province, spreading rapidly across the world, and by August 2003, over 8000 cases were reported and a fatality rate of $10 \%$ became apparent [23]. In 2007, researchers warned of the threat of coronaviruses emerging and remerging because of their ability to undergo genetic recombination [24]. These researchers wrote "that the presence of a large reservoir of SARS-CoV-like viruses in horseshoe bats, together with the culture of eating exotic mammals in southern China, is a time bomb. The possibility of the re-emergence of SARS and other novel viruses from animals or laboratories and therefore the need for preparedness should not be ignored" [24] (p. 683).

In 2012, a further novel coronavirus emerged named Middle East Respiratory Syndrome (MERS) now labelled MERS-CoV and appeared initially in Saudi Arabia [25]. However, MERS-CoV did not seem to be easily transmitted and was largely contained with only 2468 cases confirmed. Nevertheless, the symptoms which developed in those infected were reported to range from the common cold to SARS. Interestingly, the mortality rate according to the WHO is estimated to be $35 \%$ of reported patients. The most recent novel coronavirus which gripped the world emerged in Wuhan, China in December 2019, although the transfer of the virus to the human population continues to be investigated [26].

The summary of symptomatology and pathology of coronaviruses with an emphasis on SARS-CoV-2 is given in Table 1. What can be noted is that there are common

Table 1 List of known symptoms ranging from common to severe in SARS-Cov2 and post-infection pathologies identified to date

\begin{tabular}{|c|c|}
\hline Symptoms & Post-infection symptomology/pathology \\
\hline $\begin{array}{l}\text { Common }^{1} \\
\text { Fever* } \\
\text { Dry cough* }^{*} \\
\text { Dyspnea* }^{*} \\
\text { Headache } \\
\text { Dizziness } \\
\text { Generalised weakness } \\
\text { Vomiting } \\
\text { Diarrhoea }\end{array}$ & $\begin{array}{l}\text { Persistent [99] [100] [28] } \\
\text { Dyspnea } \\
\text { Fatigue } \\
\text { Headache } \\
\text { Anosmia } \\
\text { (from }>28 \text { to } 72 \text { days post-infection exac- } \\
\text { erbated by increasing age, body mass } \\
\text { index, and female) } \\
\text { Neuropsychiatric [99] [101] } \\
\text { Insomnia } \\
\text { Fatigue } \\
\text { Cognitive impairment } \\
\text { Anxiety } \\
\text { (up to } 6 \text { months post-infection) } \\
\text { Multi-organ impairment [102] [103] } \\
\text { Heart (mild systolic dysfunction and } \\
\text { myocarditis), lung, liver, pancreas } \\
\text { Gastro-intestinal [104] } \\
\text { Loss of appetite } \\
\text { Nausea } \\
\text { Acid reflux } \\
\text { Diarrhoea }\end{array}$ \\
\hline $\begin{array}{l}\text { Severe [105] } \\
\text { Hypoxia } \\
\text { Delirium } \\
\text { Encephalopathy } \\
\text { Hypercoagulability } \\
\text { Pulmonary fibrosis } \\
\text { Death }\end{array}$ & \\
\hline
\end{tabular}

*Similar to SARS and MERS. "Commonly associated with Long COVID Syndrome 
symptoms across SARS, MERS, and SARS-CoV-2. However, over the course of the pandemic, which is now approaching an 18 month period, data are emerging which indicate that there are more severe symptoms which can lead to death. In addition, there is now strong evidence, indicating that there are persistent symptoms and pathology that develop for a period of up to 6 months post-infection in some people, commonly referred to as long-COVID [27].

This brief summary of the emergence of coronaviruses along with the symptoms and pathology underscores the unpredictability with respect to the emergence of the virus as different strains, and the range of symptoms which can be experienced. Although vaccines are now available for SARS-CoV-2, containment and eradication should still be considered a strategy as the re-emergence of the virus does not guarantee that the human population will be protected from a new strain. In addition to containment and eradication, strategies to mitigate disease severity from future coronaviruses should also be highly advised.

\section{SARS-CoV-2 and existing health status}

It has been suggested that the world has been living with invisible pandemics such as obesity, sedentary behaviour, diabetes, and other non-communicable diseases which now appear to be colliding with SARS-CoV-2 [28]. If this is the case, then the additional complexity with respect to symptoms and pathology of SARS-CoV-2 are likely related to the existing health status of the individual.

The emerging data suggest that the most severely affected are those with pre-existing comorbidities such as dyspnea, chronic obstructive pulmonary disease (COPD), cardiovascular disease, and hypertension, which can lead to a higher risk of severe illness and intensive-care unit admission [29]. Other comorbidities which could progress the disease include metabolic disorders such as Type 2 diabetes mellitus (T2DM), hypo- or hyperthyroidism, and dyslipidaemia [30]. Very recent evidence suggests that SARS-CoV-2 severity and mortality might be due to the associated virus-activated "cytokine storm syndrome" [31] or a chronic inflammatory environment associated with these comorbidities. Specifically, cytokines such as Interleukin-6 (IL-6) and the acute phase C-reactive protein (CRP) are elevated in patients with severe SARS-CoV-2 [32]. This is also the case with T2DM [33]. Importantly, there seems to be a potentially critical role for body mass or adiposity with inflammation and the incidence of severe pneumonia, which now seems to be a complication in the end stage of SARS-CoV-2 [30]. Therefore, the impaired metabolic health associated with high adiposity could be a risk factor for increasing the severity of SARS-CoV-2. Added to this is the real possibility that existing non-infectious comorbidities are superimposed on the SARS-CoV-2 pandemic, which pose a potentially enormous global population health risk until worldwide immunity occurs. A recent suggestion has also raised the question as to whether the pandemic will have a negative effect on population health given that many communities have been unable to leave their homes and engage in their normal activities (work, school, fitness facilities, parks, and playgrounds), whereby if this becomes the norm, it might lead to increased sedentary behaviour and less healthy lifestyles [28].

Given the propensity for morbidity and mortality with SARS-CoV-2, and the severity of symptoms likely linked to presence of low-grade systemic inflammation, future works need to explore viable interventions that may target inflammation, and thus reduce symptoms of SARS-CoV-2 and the risk of associated complications. As such, the reduction in population and individual vulnerability through alleviation of comorbidities might be critical for both reduced disease severity, mortality, and herd immunity.

To this end, it has been well established that acute moderate-intensity exercise is capable of producing anti-inflammatory effects through secretion of myokines from the contracting skeletal muscle [34]. Furthermore, engagement in a long-term exercise has been shown to reduce chronic levels of CRP and tumour necrosis factor-alpha (TNF- $\alpha$ ) [35]. Inflammatory cytokines produced during acute inflammation are responsible for a diverse range of signalling events within cells. These anti-inflammatory effects are also applicable to clinical population groups, reducing the inflammatory state and the risk of disease or associated symptoms related to the cardio-metabolic, auto-immune, and respiratory systems.

Overall, the emerging data and literature suggest that SARS-CoV-2 severity could be attenuated in individuals with a better inflammatory milieu [36] which can be achieved through improving healthy lifestyle behaviours across the lifespan potentially protecting individuals and communities from non-communicable diseases and viral infection [37]. For instance, it has recently been reported in a sample of 48,440 individuals who have been infected with SARS-CoV-2 that the odds of death were 2.49 times greater for those consistently inactive compared with individuals meeting the minimum physical activity guidelines [38], which suggests that habitual physical activity could play a role in ameliorating the disease trajectory.

It is yet to be directly and empirically investigated whether habitual exercisers have improved immunity with respect to SARS-CoV-2. There are, however, some promising data from a Brazilian study which reports that sufficient physical activity levels are associated with a lower prevalence of COVID-19-related hospitalizations with at least 150 min per week of moderate-intensity, or 75 min a week of vigorous-intensity physical activity potentially reducing 
this prevalence by $34.3 \%$ [18], 39. Thus, the mechanism by which either a chronically higher inflammatory environment, or conversely, an attenuated one could alter disease severity or progression needs urgent development and investigation as this could be a means to future-proof the health system and potentially mitigate disease severity and mortality should a similar zoonotic disease emerge.

\section{Characteristics of SARS-CoV-2 and the immune response}

The morphology of SARS-CoV-2 is well reported with the spike or S-glycoprotein a key functional aspect of the virus. The spike protein presents two subtypes responsible for invasion into human cells, S1 and S2. In S1, the receptor-binding domain (RBD) interacts with angiotensin-converting enzyme-2 (ACE2) on the host cell, whereas the S2-binding domain is responsible for virus-cell membrane fusion and viral entry with higher affinity [40]. Interestingly, the ACE2 is present in the epithelial cells of the lung and in areas such as the heart, kidneys, gastro-intestinal tract, liver, and bladder, with adults generally demonstrating a higher expression of ACE2 [41].

Figure 1 depicts the cascade of events that are thought to occur when infected with SARS-CoV-2. Upon viral entry via the naso-oral cavity, the ACE2 receptor engages the RBD of SARS-CoV-2 to initiate replication [42]. Binding is detected by Toll-like receptor-7 (TLR-7), which then orchestrates the cascade of inflammatory cytokines, with disease severity hallmarked by IL-2, IL-17, Interferon (IFN-y) inducible protein 10, granulocyte-colony stimulating factor (GCSF), macrophage inflammatory protein-1 alpha (MIP-1a), monocyte chemoattractant protein-1 (MCP-1), and TNF-a [43]. During viral infections, $\mathrm{T}$ cells play a key regulatory role, with CD4 cells aiding B cells in antibody production and the

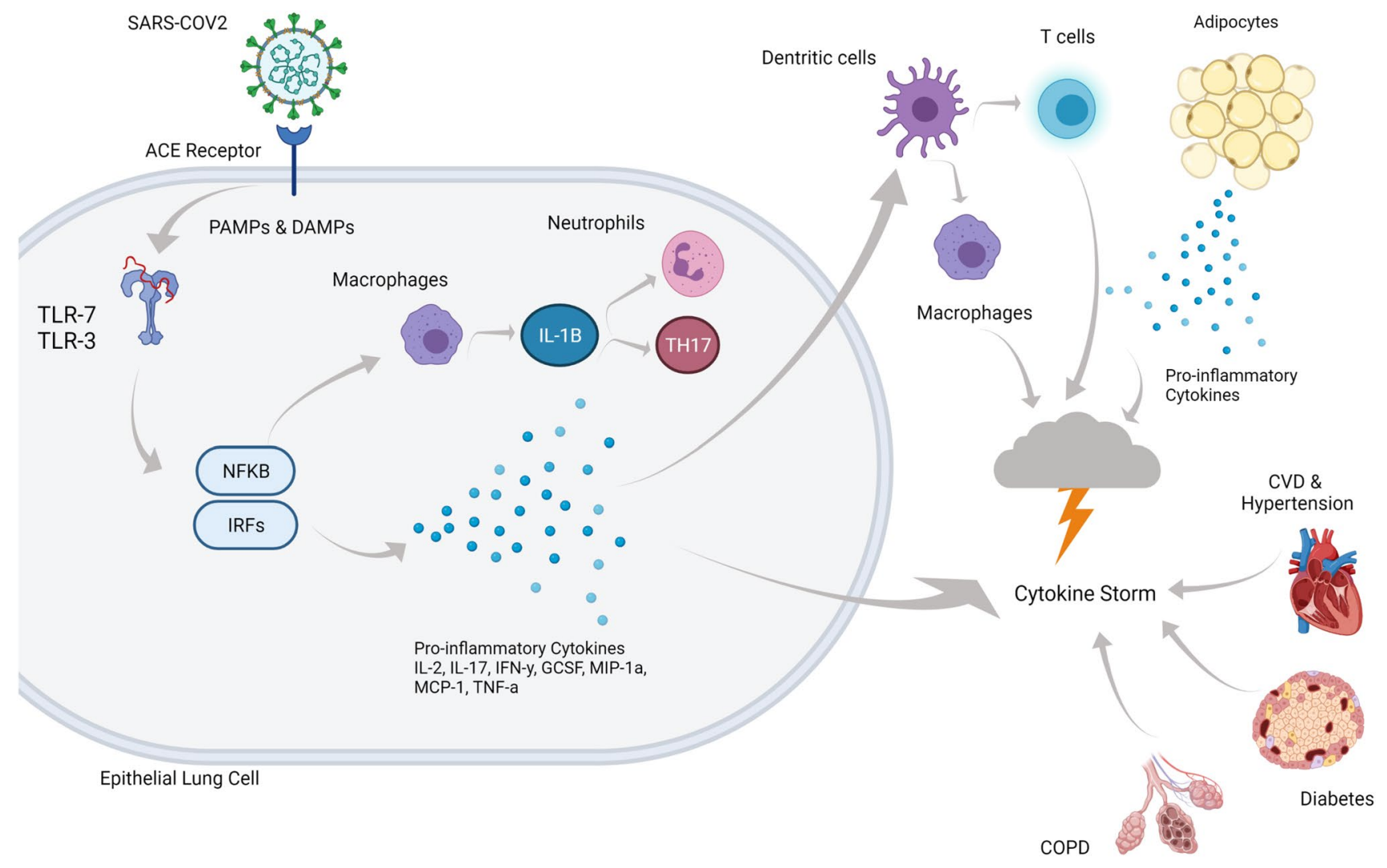

Fig. 1 As the SARS-CoV2 virus enters the respiratory system, it uses spike glycoprotein (S-protein) to neutralize antibodies and bind to the receptors of the host cell via 2 sub-units, S1 and S2. S1 initiates an immune response through binding to the angiotensin-converting enzyme-2 (ACE2) receptor while S2 allows entry into the cell. Initiation of the immune response uses pattern recognition receptors (PRRs) to recognise associated molecular patterns (PAMPs) and Damage-Associated Molecular Pattern (DAMPs). Binding of SARSCOV2 to ACE2 receptor initiates a cascade of responses including binding to Toll-like receptor (TLR)-3 and 7 and nuclear-factor kappa-
$\mathrm{B}$ (NFKB) and interferon-regulatory factors (IRFs) instigate release of pro-inflammatory cytokines and macrophages, neutrophils, and T-helper 17 (TH17). Dendritic cells respond to the inflammatory response, activating $\mathrm{T}$ cells, and macrophages, all contributing to the cytokine storm. Presence of adipocytes secreting pro-inflammatory cytokines in addition to inflammation associated with pre-existing conditions such as cardiovascular disease (CVD), hypertension, chronic obstructive pulmonary disease (COPD), and diabetes may exacerbate the cytokine storm, exacerbating symptoms, and increasing risk of hospitalisation and death. Created with BioRender.com 
signalling of other immune cells, whereas CD8 cells destroy infected cells to lower the viral burden [42]. However, once infected with SARS-CoV-2, epithelial lung cells produce IL-8 which then acts as a chemoattractant for neutrophils and T-lymphocytes. Consequently, exposure to persistent inflammatory signals is associated with the chronic activation of T cells and T-cell exhaustion [44], whereby a decline in function is observed. Lucas et al. [45] analysed peripheral blood mononuclear cells and plasma samples from those with moderate-to-severe SARS-CoV-2, and demonstrated substantial reductions in CD4 and CD8 T cells and increased expression of monocytes, low-density neutrophils, and eosinophils. T-cell exhaustion may then be responsible for the cytokine storm, suppression of T cells, B cells, and NK cells, as well as immune dysregulation. Identifying distinguishing characteristics of immune dysregulation may elucidate the underpinning mechanisms for severe and fatal cases of SARS-CoV-2 and indicate a management intervention.

\section{SARS-CoV-2 and the cytokine storm}

The 'Cytokine Storm' is a common term used to describe the extreme and complex inflammatory response to a viral infection, otherwise known as secondary hemophagocytic lymphohistiocytosis (sHLsH), or macrophage activation syndrome (MAS) [46]. The cytokine storm during SARS$\mathrm{CoV}-2$ has been particularly characterised by high levels of ferritin and pro-inflammatory cytokines [47], and elevated D-dimer [48], and can be associated with intravascular coagulation [49]. In a normal viral response, an antigen is recognised which activates the adaptive and innate immunity. The immune response results in the release of both pro- and anti-inflammatory cytokines and chemokines. For various reasons (e.g., underlying conditions, previous exposure to stress, etc.), though many remain unknown, the inflammatory response is disproportionate to the viral load and results in hypercytokinemia, leading to acute respiratory distress syndrome (ARDS), multi-organ failure, and, in extreme cases, death [49]. Given the rapidly developing research and reporting surrounding SARS-CoV-2, at the time of writing, the cytokine storm remains a popular hypothesis for severe infections which tend to lead to mortality, although others such as the bradykinin hypothesis have also recently been suggested [50].

In SARS-CoV-2, the underlying mechanisms of the cytokine storm are not fully understood and, in a recent paper [51], have been questioned. Nevertheless, impaired initial response of type-1 IFNs has been associated with severe SARS-CoV-2 [52] in addition to lymphocytopenia [48]. Moreover, autopsy results have shown specific atrophy in the spleen and other secondary lymphoid organs [53]. These impairments may account for a reduction in lymphocytes (e.g., CD4 and CD8 T cells) that are necessary for fighting off infection [53]. Indeed, it has been shown that peripheral blood lymphocyte cell counts of CD3, CD4, and CD8 were all attenuated with greater severity of disease [48]. In addition to reduced lymphocyte function, monocytes and macrophages are found to be upregulated [54]. Macrophages release cytokines and, when dysregulated, increase the propensity for the elevated cytokine response [55]. The primary cytokines that result in the cytokine storm include TNF-alpha, IL-6, IL-8, and IL-10, among others [52]. It is proposed that elevated levels of these cytokines can lead to increased viscosity of the blood [46] which can further increase the possibility of thromboembolism or vascular coagulation.

There are several pre-disposing mechanistic factors that increase the likelihood of the cytokine storm occurring secondary to SARS-CoV-2 infections, such as inefficient identification and clearing of the viral antigen and inadequate activation of interferons, epigenetic dysregulation of ACE2, and interferon-regulated genes [56], among several others [46]. Importantly, it is believed that the risk of mortality is greatly increased with underlying risk factors such as smoking and comorbidities such as T2DM, obesity, metabolic disease, and cardiovascular disease. The reason for this increased risk is likely due to one or a combination of the pre-disposing mechanistic factors at play in these conditions. These will be further described in detail below; however, the reader is reminded that in many instances, development of these conditions, and consequently, severe SARS-CoV-2 may be modulated by lifestyle.

\section{Comorbidities that increase the risk of severe SARS-CoV-2 infection}

While not an exhaustive list, the following section will highlight four comorbidities that are now reported and identified as potential to increase the severity of SARS-CoV-2 and the associated inflammatory profiles. They include: (1) hypertension, (2) cardiovascular disease, (3) obesity, and (4) diabetes mellitus. Following discussion of these conditions, the remainder of the review aims to highlight the impact of lifestyle modification — notably exercise and physical activityand its potential to ameliorate the inflammatory response and associated severity of the current SARS-CoV-2. These lifestyle modifications are particularly important to reduce population-wide severity of future coronavirus pandemics that are expected to occur.

\section{i. Hypertension and cardiovascular morbidity}

Hypertension (HTN) and cardiovascular morbidity have been identified as two underlying or preexisting conditions that can increase the severity of 
SARS-CoV-2 disease [57]. A recent meta-analysis of 29,750 patients identified that the prevalence of HTN and cardiovascular disease (CVD) in SARS-CoV-2 patients was $32.7 \%$ and $18.2 \%$, respectively. Although this study reported that neither pre-existing HTN nor CVD was associated with greater mortality, they did report that CVD was associated with greater levels of invasive mechanical ventilation [58]. In contrast, earlier studies have reported that poor outcomes are significantly greater for HTN and CVD patients suffering from SARS-CoV-2 [59], with one suggesting the case-fatality rate to be double for HTN and over triple for CVD [60].

The mechanisms underlying disease severity in cases with HTN and CVD are not entirely clear. It is suggested that underlying CVD can be exacerbated by myocyte damage caused by hypoxia, in addition to the cytokine storm discussed above [58]. Further to this, people with HTN are often on anti-hypertensive medication such as ACE inhibitors and angiotensin receptor blockers. Such medications can inhibit the renin-angiotensin-aldosterone system which may increase the vulnerability of individuals with HTN to severe SARS-CoV-2 infection [59]. Whether medications increase ACE2 expression on the cell surface or decrease ACE2 expression, the susceptibility of the individual to the virus is maintained and can lead to severe infection [59].

In the absence of severe SARS-CoV-2 disease, both CVD and HTN have been associated with immune dysregulation and meta-inflammation. CVD is characterised by chronic inflammation where innate immune system activation results in cardiac damage [61] or responds to the rupture of plaque within the vessel in increases of endothelial dysfunction [61, 62]. The immunologic mechanism for HTN begins in the vascular wall, where increases in blood pressure promote oxidative damage causing upregulation of dangerassociated molecular patterns (DAMPs) and hypertensive-specific neoantigens. A further cascade through Toll-like receptors and dendritic cells leads to production of inflammatory cytokines and chemokines, while B cell and $\mathrm{T}$ cells can promote effector $\mathrm{T}$ cells to accumulate in blood vessels, the heart and kidneys [63]. Further increases in blood pressure augment this response, leading to vascular stiffness, fibrosis, and dysfunction [64]. Each of these can lead to structural changes in the vasculature, and in the heart, ultimately leading to myocardial infarction or heart failure in extreme cases. Mitigating the chronic inflammation associated with these conditions can attenuate overall risk of developing HTN or CVD and also the severity of disease if SARS-CoV-2 is contracted.

\section{ii. Obesity and diabetes mellitus}

While the risk of fatal outcome from contracting SARS-CoV-2 is reported to be higher in those with T2DM [65], recent literature suggests that the presence of diabetes does not increase the likelihood of infection; rather, it will influence the course of the infection [66]. Immune dysregulation reported in metabolic conditions such as T2DM includes dysfunction of NK cells, B cells, T cells, and cytokine signalling pathways [65]. Given the immune dysregulation in T2DM, it is not surprising that both Type 1 and Type 2 diabetes mellitus were independently associated with increased odds of in-hospital fatality from SARS-CoV-2 [67]. In support, it has also been reported that T2DM patients demonstrate a higher inflammatory response, higher neutrophils, and CRP with lower lymphocyte and eosinophil counts, and a more aggressive course of the disease [68]. Similarly, obese individuals demonstrate insulin resistance, elevated systemic inflammation, and over activity of the RAAS [69]. For example, the pathophysiological traits of obesity, such as elevated adiposity and associated inflammation, increase the susceptibility to more severe disease outcomes [70]. The specific physiological reason for this observation is not entirely clear. However, as already outlined, adults generally demonstrate higher expression of ACE2, and given the apparent higher expression of ACE2 in adipose tissue, it is highly possible that adipose tissue might act as a viral reservoir for SARS-CoV-2 infection [71, 72]. Therefore, obesity presents as a significant risk factor for infection and disease severity. As such, lifestyle modifications associated with reducing adiposity and associated inflammation may be favourable in ameliorating the severity of SARS-Cov2 [73].

In metabolic disease such as T2DM and obesity, adipose tissue exhibits a regulatory role in cytokine release; termed adipokines, the cytokines released from adipocytes are involved in the aetiology of diabetes, insulin resistance, and the development of atherosclerosis [74]. Particularly, those who present with T2DM demonstrate an exaggerated inflammatory profile/cytokine expression [75]. Notably, the presence of TNF- $\alpha$ in T2DM may prime neutrophils, and this excessive activation of neutrophils may contribute to inflammatory injury (that is, ARDS) [76]. In normal immune reactions, the priming of neutrophils regulates the intensity of the immune response. However, in conditions such as T2DM, where elevated immune-inflammatory markers are a key pathological characteristic, the presence of TNF- $\alpha$ may enhance the neutrophil response and may aid in the pathogenesis of inflammatory-related manifestations such as ARDS and inflammatory tissue injury. Furthermore, 
neutrophilia is predictive of poor patient outcomes, with the neutrophil-lymphocyte ratio an independent risk factor for severe disease outcomes [77].

\section{Habitual physical activity on meta-inflammation for SARS-CoV-2 defence}

Meta-inflammation refers to the low-grade, chronic inflammatory state that is present in many chronic health conditions, such as obesity, hypertension, cardiovascular disease, and other metabolic disorders [78]. Positive benefits are associated with chronic resistance and/or aerobic exercise training which attenuates meta-inflammation in both women and men in such conditions, and with increasing age [79]. However, a recent meta-analysis looking at the effects of aerobic exercise on TNF- $\alpha$, IL-6, and CRP markers in middle-to-older aged adults suggested that further randomized controlled trials should be undertaken to determine the effect of aerobic exercise on markers other than those listed above, and strengthen the evidence for or against attenuating
IL-6. Importantly, while the SARS-CoV-2 cytokine storm is known to involve many different types of inflammatory markers, it is widely perceived that the presence of 'metainflammation', typically marked by elevated levels of TNF$\alpha$, IL-6, CRP, and plasminogen activator inhibitor (PAI-1) due to greater levels of adipose tissue and adipokines, predisposes an individual to greater risk and severity of the disease. Therefore, mitigating meta-inflammation through habitual exercise could be a particularly important defence mechanism against SARS-CoV-2.

In the previous section, we briefly outlined the relationship that comorbidities (HTN, CVD and obesity, and diabetes mellitus) have with the risk of severe SARS-CoV-2 based on the inflammatory consequences of these conditions. Here, we outline the known benefits of exercise on the inflammatory responses in these comorbidities with respect to improving the inflammatory profile as a first line of defence for ameliorating the chronic disease, and in so doing reducing the likelihood of severe symptoms of SARS-CoV-2 if infected. Figure 2 is a representation of the benefits and the intervention that exercise is thought to have in conferring a level of protection against severe COVID-19.

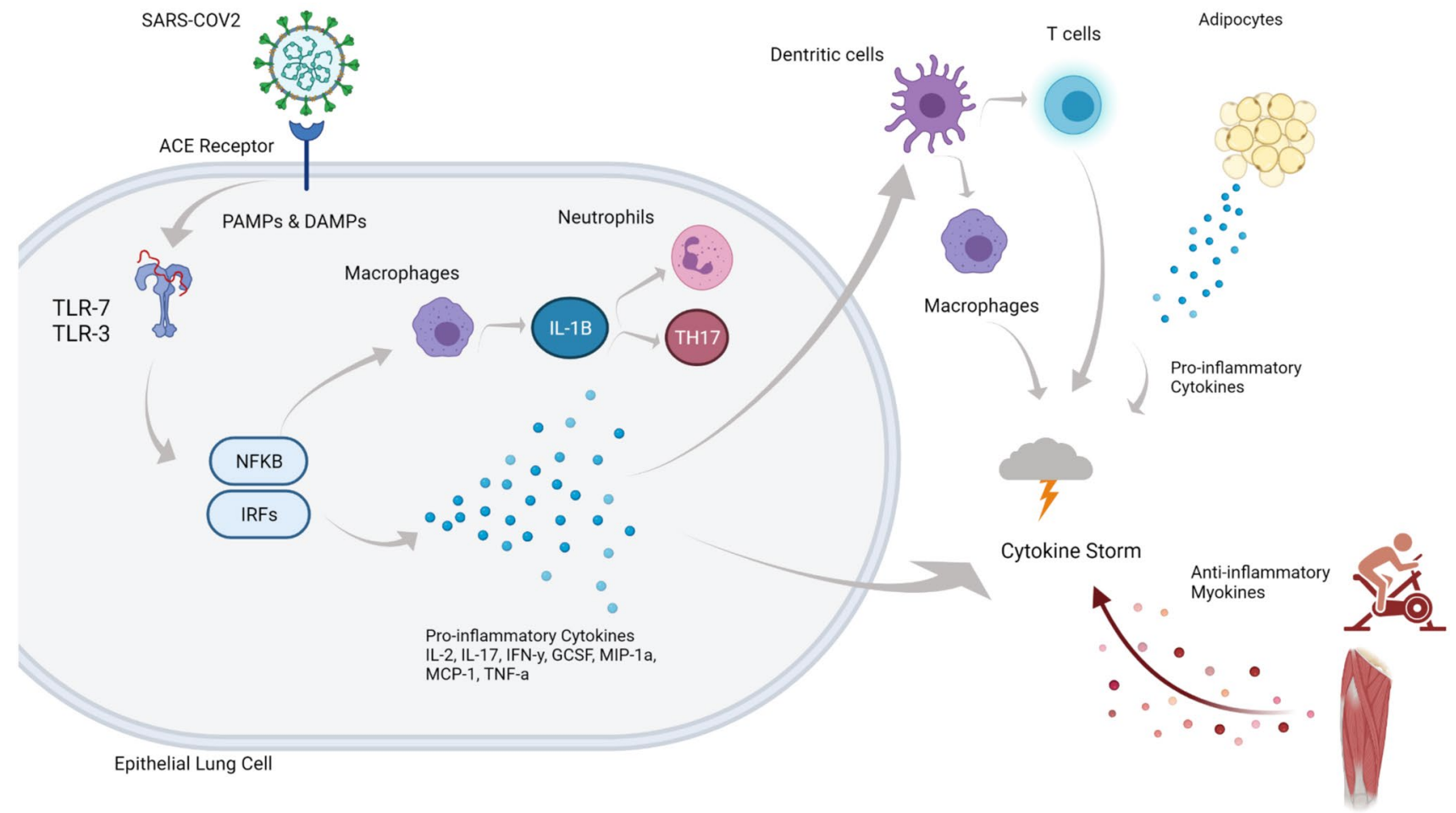

Fig. 2 Following the SAR-COV2 entering the respiratory system, the cascade of events as outlined in Fig. 1 proceeds, including secretion of pro-inflammatory cytokines, initiation of macrophages and response from dendritic cells, all of which contribute to the development of a cytokine storm, with the presence of high adiposity (obesity) or pre-existing conditions increasing risk of hospitalisation or death. As outlined above, lower adiposity reduces the secretion of pro-inflammatory cytokines which contribute to the storm. Furthermore, regular engagement in physical activity and exercise reduces risk of comorbidities and facilitates the release anti-inflammatory myokines due to muscle contraction, also reducing the likelihood of a cytokine storm following infection with SARS-COV2. Created with BioRender.com 
It has been well documented in humans and animal models that moderate, habitual exercise can improve the inflammation and oxidative stress in HTN. For example, the progression of HTN in young spontaneously hypertensive rats was investigated with moderate-intensity exercise over a 16 week period [80]. As expected, chronic exercise in hypertensive rats resulted in reduced blood pressure and cardiac hypertrophy with improved diastolic function. Importantly, these modifications were also accompanied by significant decreases in both myocardial and circulating pro-inflammatory cytokines TNF- $\alpha$ and IL- $1 \beta$. In humans, the pro-inflammatory cytokines interleukin IL-6, IL-1 $\beta$, and TNF- $\alpha$ are elevated with increasing blood pressure and hypertension [81]. Although a range of pharmacological interventions reduce HTN and can have favourable outcomes with respect to mortality and cardiovascular incidence rates [82], pharmacological interventions appear to have a limited effect on the level of inflammation associated with either HTN or CVD, possibly because the benefits derived from anti-hypertensive drugs are largely specific, e.g., diuretics reduce the incidence of stroke and heart failure but not the incidence of myocardial infarction or predisposition to the development of T2DM [83].

In contrast, either acute or habitual exercise as a therapy has been shown to improve and reduce the progression of HTN through a multifactorial process which includes improved autonomic control [84], modulation of catecholamine response [85], and decreases in vascular resistance [86]. These physiological adaptations are also accompanied by improved pro-inflammatory biomarkers such as CRP in hypertensive individuals [87]. The improved blood pressure and cardiovascular function which is accompanied by more favourable inflammatory biomarkers places exercise as a powerful option in the defence against the threat of a cytokine storm that would be superimposed by SARS-CoV-2 infection.

There is now good evidence that both aerobic and resistance exercise training or a combination improves the fat-free mass for both men and women after 10 weeks of training, albeit that there is a biased dose-response relationship between men and women; a higher fat-free mass gain for men [88]. In a study comparing resistance, aerobic, and control participants over 10 weeks, exercise training was not found to reduce IL-6 [89]. However, these authors did find that resistance and aerobic exercise individually reduced CRP by $32.8 \%$ and $16.1 \%$, respectively from baseline. Although at baseline, CRP was positively correlated with IL-6, fat mass, and intra-abdominal mass and was inversely correlated with aerobic fitness measures. However, a salient finding was that adiposity was differentially reduced for both exercise groups (intra-abdominal fat for aerobic vs. total body fat mass for resistance training). The totality of these findings suggests that reductions in adiposity by either aerobic or resistance training may improve the inflammatory profile, since higher adiposity is also associated with inflammatory cytokine regulation [90].

We have also previously reported that habitual exercise ameliorates chronic inflammation in T2DM [91] a candidate comorbidity for severe SARS-CoV-2. In this study, 2 months of moderate exercise, 3 days per week resulted in a reduction in chronic inflammation in such markers as IL-6 and CRP in previously sedentary middle-aged men. Interestingly, our additional study suggests that it is even more critical to increase physical activity and reduce adiposity and associated inflammatory response for the Indigenous population [92]. This is particularly important given that there is emerging evidence that ethnicity may also be a factor in contracting SARS-CoV-2 [93], which may be influenced by potential biological and socio-economic factors. To that end, recent analyses of hospital admissions indicate that access to care is a key determinant of vulnerability, since outcomes were not different between ethnic groups; rather, age, sex, insurance, and existing comorbidities were associated with higher risk of all-cause-inhospital mortality [94].

\section{Conclusion}

In summary, acute and habitual physical activity is associated with reduced adiposity and thus reduces the likelihood of systemic inflammation. Habitual exercise is known to improve metabolic, cardiovascular, and cardiorespiratory capacities which provides resilience in the face of viral threats such as SARS-CoV-2. The emerging evidence indicates that the risk of SARS-CoV-2-related complications and mortality appears to be higher in individuals with comorbidities, and/or evidence of systemic inflammation. For this reason, engagement in habitual physical activity and exercise should be highly recommended as a strategy to improve the immune system and its response, thereby potentially reducing the risk of symptoms and lifethreatening complications if infected.

Author contributions FM coordinated the research team and provided the structure for the manuscript. All members contributed equally to writing and analysis of published research.

Funding Funding for this review was received from a Charles Sturt University COVID-19 Research Grant.

Availability of data and materials Not applicable.

Code availability Not applicable. 


\section{Declarations}

Conflict of interest The authors declare that there are no conflicts of interest.

Ethics approval Not applicable.

Consent to participate Not applicable.

Consent for publication All authors consent to the submission and publication of the manuscript if accepted.

\section{References}

1. Mahase E. Coronavirus covid-19 has killed more people than SARS and MERS combined, despite lower case fatality rate. BMJ. 2020;368:m641.

2. World Health Organisation. Naming the coronavirus disease (COVID-19) and the virus that causes it. https://www.who.int/ emergencies/diseases/novel-coronavirus-2019/technical-guida nce/naming-the-coronavirus-disease-(covid-2019)-and-thevirus-that-causes-it\#: :text=Viruses\%20are\%20named\%20bas ed\%20on,Viruses\%20(ICTV). Accessed 12 July 2021.

3. World Health Organisation. Data dashboard. https://covid19.who. int/table. Accessed 20 October 2021.

4. Baker MG, Wilson N, Anglemyer A. Successful elimination of Covid-19 transmission in New Zealand. N Engl J Med. 2020;383:e56.

5. Iwasaki A, Wong P. The immune havoc of COVID-19. Sci Am. 2021;324:31-7.

6. Rodpothong P, Auewarakul P. Viral evolution and transmission effectiveness. World J Virol. 2012;1:131-4.

7. Oliu-Barton M, Pradelski BSR, Aghion P, Artus P, Kickbusch I, Lazarus JV, Sridhar D, Vanderslott S. SARS-CoV-2 elimination, not mitigation, creates best outcomes for health, the economy, and civil liberties. Lancet. 2021;397:2234-6.

8. Zhang S. A vaccine reality check. The Atlantic 2020;

9. Goldenberg MJ. Vaccine hesitancy: public trust, expertise, and the war on science. Pittsburgh: University of Pittsburgh Press; 2021.

10. Taylor A. Steer clear of needles: bad ads could 'disgust' people, turn them off COVID-19 vaccines. Sydney Morning Herald 2021;

11. Centre for Disease Control. CDC recommends use of Johnson \& Johnson's Janssen COVID-19 vaccine resume. https://www. cdc.gov/coronavirus/2019-ncov/vaccines/safety/JJUpdate.html. Accessed 12 July, 2021.

12. Volz E, Mishra S, Chand M, Barrett JC, Johnson R, Geidelberg L, Hinsley WR, Laydon DJ, Dabrera G, O'Toole Á. Assessing transmissibility of SARS-CoV-2 lineage B. 1.1. 7 in England. Nature. 2021;593:266-9.

13. Potts M. My community refuses to get vaccinated. Now delta is here. The Atlantic. https://www.theatlantic.com/health/archive/ 2021/07/unvaccinated-different-anti-vax/619523/. Accessed 12 July 2021.

14. Matthews CE, Ockene IS, Freedson PS, Rosal MC, Merriam PA, Hebert JR. Moderate to vigorous physical activity and risk of upper-respiratory tract infection. Med Sci Sports Exerc. 2002;34:1242-8.

15. Yan H, Kuroiwa A, Tanaka H, Shindo M, Kiyonaga A, Nagayama A. Effect of moderate exercise on immune senescence in men. Eur J Appl Physiol. 2001;86:105-11.

16. Pedersen BK, Saltin B. Exercise as medicine-evidence for prescribing exercise as therapy in 26 different chronic diseases. Scand J Med Sci Sports. 2015;25(Suppl 3):1-72.
17. Ejaz H, Alsrhani A, Zafar A, Javed H, Junaid K, Abdalla AE, Abosalif KOA, Ahmed Z, Younas S. COVID-19 and comorbidities: deleterious impact on infected patients. J Infect Pub Hlth. 2020;13:1833-9.

18. Brawner CA, Ehrman JK, Bole S, Kerrigan DJ, Parikh SS, Lewis BK, Gindi RM, Keteyian C, Abdul-Nour K, Keteyian SJ. Inverse relationship of maximal exercise capacity to hospitalization secondary to coronavirus disease 2019. Mayo Clin Proc. 2021;96:32-9.

19. International Committee on Taxonomy of Viruses. Coronaviridae. https://talk.ictvonline.org/ictv-reports/ictv_9th_report/posit ive-sense-rna-viruses-2011/w/posrna_viruses/223/coronaviridaefigures. Accessed 12 July, 2021.

20. Tyrrell DAJ, Bynoe ML. Cultivation of a novel type of commoncold virus in organ cultures. Br Med J. 1965;1:1467.

21. Almeida JD, Tyrrell DAJ. The morphology of three previously uncharacterized human respiratory viruses that grow in organ culture. J Gen Virol. 1967;1:175-8.

22. Tyrrell DAJ, Almeida JD, Berry DM, Cunningham CH, Hamre D, Hofstad MS, Malucci L, McIntosh K. Coronaviruses. Nature. 1968;220:650.

23. Gu J, Korteweg C. Pathology and pathogenesis of severe acute respiratory syndrome. Am J Pathol. 2007;170:1136-47.

24. Cheng VC, Lau SK, Woo PC, Yuen KY. Severe acute respiratory syndrome coronavirus as an agent of emerging and reemerging infection. Clin Microbiol Rev. 2007;20:660-94.

25. World Health Organisation. Middle East respiratory syndrome coronavirus (MERS-CoV). https://www.who.int/en/news-room/ fact-sheets/detail/middle-east-respiratory-syndrome-coronavirus(mers-cov). Accessed 12 July, 2021.

26. Qiu J. Chasing plagues. Sci Am. 2020;322:20-5.

27. Sudre CH, Murray B, Varsavsky T, Graham MS, Penfold RS, Bowyer RC, Pujol JC, Klaser K, Antonelli M, Canas LS, Molteni E, Modat M, Jorge Cardoso M, May A, Ganesh S, Davies R, Nguyen LH, Drew DA, Astley CM, Joshi AD, Merino J, Tsereteli N, Fall T, Gomez MF, Duncan EL, Menni C, Williams FMK, Franks PW, Chan AT, Wolf J, Ourselin S, Spector T, Steves CJ. Attributes and predictors of long COVID. Nature Med. 2021;27:626-31.

28. Hall G, Laddu DR, Phillips SA, Lavie CJ, Arena R. A tale of two pandemics: how will COVID-19 and global trends in physical inactivity and sedentary behavior affect one another. Prog Cardiov Dis. 2021;64:108-10.

29. Jain V, Yuan J-M. Predictive symptoms and comorbidities for severe COVID-19 and intensive care unit admission: a systematic review and meta-analysis. Int J Pub Hlth. 2020;65:533-46.

30. Stefan N, Birkenfeld AL, Schulze MB, Ludwig DS. Obesity and impaired metabolic health in patients with COVID-19. Nature Rev Endocrinol E-pub ahead of print.

31. Mehta P, McAuley DF, Brown MH, Sanchez E, Tattersall RS, Manson J. COVID-19: consider cytokine storm syndromes and immunosuppression. Lancet. 2020;395:1033-4.

32. Ruan Q, Yang K, Wang W, Jiang L, Song J. Clinical predictors of mortality due to COVID-19 based on an analysis of data of 150 patients from Wuhan, China. Intens Care Med 2020; E-pub ahead of print.

33. Maddaloni E, Buzzetti R. Covid-19 and diabetes mellitus: unveiling the interaction of two pandemics. Diabet/Metabol Res Rev 2020; e33213321.

34. Steensberg A, van Hall G, Osada T, Sacchetti M, Saltin B, Pedersen BK. Production of interleukin-6 in contracting human skeletal muscles can account for the exercise-induced increase in plasma interleukin-6. J Physiol. 2000;529:237-42.

35. You T, Arsenis NC, Disanzo BL, Lamonte MJ. Effects of exercise training on chronic inflammation in obesity : current evidence and potential mechanisms. Sports Med. 2013;43:243-56. 
36. Laddu DR, Lavie CJ, Phillips SA, Arena R. Physical activity for immunity protection: inoculating populations with healthy living medicine in preparation for the next pandemic. Prog Cardio Dis. 2021;64:102-4.

37. Arena R, Lavie CJ. The global path forward-healthy living for pandemic event protection (HL-PIVOT). Prog Cardio Dis. 2021;64:96-101

38. Sallis R, Young DR, Tartof SY, Sallis JF, Sall J, Li Q, Smith GN, Cohen DA. Physical inactivity is associated with a higher risk for severe COVID-19 outcomes: a study in 48440 adult patients. $\mathrm{Br}$ J Sports Med. 2021;55:1099-105.

39. Souza FR, Motta-Santos D, dos Santos Soares D, Lima JB, Cardozo GG, Guimarães LSP, Negrão CE, dos Santos MR. Physical activity decreases the prevalence of COVID-19-associated hospitalization: Brazil EXTRA study. MedRxiv 2020;

40. Chowdhury MA, Hossain N, Kashem MA, Shahid MA, Alam A. Immune response in COVID-19: a review. J Infect Public Health. 2020;13:1619-29.

41. Yazdanpanah F, Hamblin MR, Rezaei N. The immune system and COVID-19: friend or foe. Life Sci. 2020;256:117900.

42. Mukerjee N. A brief review on the overview on immunology of COVID-19: current state of the research. Int J Sci Res. 2020;9:SR201102135538.

43. Khanmohammadi S, Rezaei N. Role of toll-like receptors in the pathogenesis of COVID-19. J Med Virol. 2021;93:2735-9.

44. Wherry EJ, Kurachi M. Molecular and cellular insights into T cell exhaustion. Nat Rev Immunol. 2015;15:486-99.

45. Lucas C, Wong P, Klein J, Castro TBR, Silva J, Sundaram M, Ellingson MK, Mao T, Oh JE, Israelow B, Takahashi T, Tokuyama M, Lu P, Venkataraman A, Park A, Mohanty S, Wang H, Wyllie AL, Vogels CBF, Earnest R, Lapidus S, Ott IM, Moore AJ, Muenker MC, Fournier JB, Campbell M, Odio CD, Casanovas-Massana A, Yale IMPACTT Team, Herbst R, Shaw AC, Medzhitov R, Schulz WL, Grubaugh ND, Dela Cruz C, Farhadian S, Ko AI, Omer SB, Iwasaki A. Longitudinal analyses reveal immunological misfiring in severe COVID-19. Nature. 2020;584:463-9.

46. Soy M, Keser G, Atagündüz P, Tabak F, Atagündüz I, Kayhan S. Cytokine storm in COVID-19: pathogenesis and overview of anti-inflammatory agents used in treatment. Clin Rheumatol. 2020;39:2085-94

47. Gómez-Pastora J, Weigand M, Kim J, Wu X, Strayer J, Palmer AF, Zborowski M, Yazer M, Chalmers JJ. Hyperferritinemia in critically ill COVID-19 patients-is ferritin the product of inflammation or a pathogenic mediator. Clin Chim Acta. 2020;509:249-51.

48. Song JW, Zhang C, Fan X, Meng FP, Xu Z, Xia P, Cao WJ, Yang T, Dai XP, Wang SY, Xu RN, Jiang TJ, Li WG, Zhang DW, Zhao P, Shi M, Agrati C, Ippolito G, Maeurer M, Zumla A, Wang FS, Zhang JY. Immunological and inflammatory profiles in mild and severe cases of COVID-19. Nat Commun. 2020;11:3410.

49. Jose RJ, Manuel A. COVID-19 cytokine storm: the interplay between inflammation and coagulation. Lancet Respir Med. 2020;8:e46-7.

50. Roche JA, Roche R. A hypothesized role for dysregulated bradykinin signaling in COVID-19 respiratory complications. FASEB J. 2020;34:7265-9.

51. Leisman DE, Ronner L, Pinotti R, Taylor MD, Sinha P, Calfee CS, Hirayama AV, Mastroiani F, Turtle CJ, Harhay MO, Legrand $\mathrm{M}$, Deutschman CS. Cytokine elevation in severe and critical COVID-19: a rapid systematic review, meta-analysis, and comparison with other inflammatory syndromes. Lancet Respir Med. 2020;8:1233-44

52. Kim JS, Lee JY, Yang JW, Lee KH, Effenberger M, Szpirt W, Kronbichler A, Shin JI. Immunopathogenesis and treatment of cytokine storm in COVID-19. Theranostics. 2021;11:316-29.

53. Xu Z, Shi L, Wang Y, Zhang J, Huang L, Zhang C, Liu S, Zhao P, Liu H, Zhu L. Pathological findings of COVID-19 associated with acute respiratory distress syndrome. Lancet Respir Med. 2020;8:420-2.

54. Chua RL, Lukassen S, Trump S, Hennig BP, Wendisch D, Pott F, Debnath O, Thürmann L, Kurth F, Völker MT. COVID19 severity correlates with airway epithelium-immune cell interactions identified by single-cell analysis. Nat Biotechnol. 2020;38:970-9.

55. Merad M, Martin JC. Pathological inflammation in patients with COVID-19: a key role for monocytes and macrophages. Nat Rev Immunol. 2020;20:355-62.

56. Sawalha AH, Zhao M, Coit P, Lu Q. Epigenetic dysregulation of ACE2 and interferon-regulated genes might suggest increased COVID-19 susceptibility and severity in lupus patients. Clin Immunol. 2020;215:108410.

57. Matsushita K, Ding N, Kou M, Hu X, Chen M, Gao Y, Honda Y, Zhao D, Dowdy D, Mok Y, Ishigami J, Appel LJ. The Relationship of COVID-19 Severity with cardiovascular disease and its traditional risk factors: a systematic review and meta-analysis. Glob Heart. 2020;15:64.

58. Chakinala RC, Shah CD, Rakholiya JH, Martin M, Kaur N, Singh H, Okafor TL, Nwodika C, Raval P, Yousuf S. COVID-19 outcomes amongst patients with pre-existing cardiovascular disease and hypertension. Cureus 2021; 13:

59. Lippi G, Wong J, Henry BM. Hypertension and its severity or mortality in Coronavirus Disease 2019 (COVID-19): a pooled analysis. Pol Arch Int Med. 2020;130:304-9.

60. Wu Z, McGoogan JM. Characteristics of and important lessons from the coronavirus disease 2019 (COVID-19) outbreak inchina: summary of a report of 72314 cases from the chinese center for disease control and prevention. JAMA. 2020;323:1239-42.

61. Frostegård J. Immunity, atherosclerosis and cardiovascular disease. BMC Med. 2013;11:1-13.

62. Jaén RI, Val-Blasco A, Prieto P, Gil-Fernández M, Smani T, López-Sendón JL, Delgado C, Boscá L, Fernández-Velasco M. Innate immune receptors, key actors in cardiovascular diseases. Basic Trans Sci. 2020;5:735-49.

63. Drummond GR, Vinh A, Guzik TJ, Sobey CG. Immune mechanisms of hypertension. Nature Rev Immunol. 2019;19:517-32.

64. Harrison DG. The immune system in hypertension. Trans Am Clin Climatol Assoc. 2014;125:130.

65. Vas P, Hopkins D, Feher M, Rubino F, Whyte M. Diabetes, obesity and COVID-19: a complex interplay. Diab, Obes Metabol. 2020;22:1892-6.

66. Apicella M, Campopiano M C, Mantuano M, Mazoni L, Coppelli A, Del Prato S. COVID-19 in people with diabetes: understanding the reasons for worse outcomes. Lancet Diab Endocrinol 2020;

67. Barron E, Bakhai C, Kar P, Weaver A, Bradley D, Ismail H, Knighton P, Holman N, Khunti K, Sattar N. Associations of type 1 and type 2 diabetes with COVID-19-related mortality in England: a whole-population study. Lancet Diab Endocrinol. 2020;8:813-22.

68. Soliman A, Nair AP, Al Masalamani MS, De Sanctis V, Khattab MAA, Alsaud AE, Sasi S, Ali EA, Hassan OA, Iqbal FM. Prevalence, clinical manifestations, and biochemical data of type 2 diabetes mellitus versus nondiabetic symptomatic patients with COVID-19: a comparative study. Acta Biomed. 2020;91:e2020010.

69. Sanchis-Gomar F, Lavie CJ, Mehra MR, Henry BM, Lippi G. Obesity and outcomes in COVID-19: when an epidemic and pandemic collide. Mayo Clin Proc. 2020;95:1445-53.

70. Chiappetta S, Sharma AM, Bottino V, Stier C. COVID-19 and the role of chronic inflammation in patients with obesity. Int $\mathrm{J}$ Obes. 2020;44:1790-2.

71. Kruglikov IL, Scherer PE. Therole of adipocytes and adipocyte-like cells in the severity of COVID-19 infections. Obesity. 2020;28:1187-90. 
72. Sharma A, Garg A, Rout A, Lavie CJ. Association of obesity with more critical illness in COVID-19. Mayo Clin Proc. 2020;95:2040-2.

73. Carter SJ, Baranauskas MN, Fly AD. Considerations for obesity, vitamin D, and physical activity amid the COVID-19 pandemic. Obesity. 2020;28:1176-7.

74. Hayashino Y, Jackson JL, Hirata T, Fukumori N, Nakamura F, Fukuhara S, Tsujii S, Ishii H. Effects of exercise on C-reactive protein, inflammatory cytokine and adipokine in patients with type 2 diabetes: a meta-analysis of randomized controlled trials. Metabol. 2014;63:431-40.

75. Guo W, Li M, Dong Y, Zhou H, Zhang Z, Tian C, Qin R, Wang $\mathrm{H}$, Shen $\mathrm{Y}, \mathrm{Du} \mathrm{K}$. Diabetes is a risk factor for the progression and prognosis of COVID-19. Diab/Metabol Res Rev. 2020;36:e3319.

76. Condliffe AM, Kitchen E, Chilvers ER. Neutrophil priming: pathophysiological consequences and underlying mechanisms. Clin Sci. 1998;94:461-71.

77. Barnes BJ, Adrover JM, Baxter-Stoltzfus A, Borczuk A, CoolsLartigue J, Crawford JM, Daßler-Plenker J, Guerci P, Huynh C, Knight JS, Loda M, Looney MR, McAllister F, Rayes R, Renaud S, Rousseau S, Salvatore S, Schwartz RE, Spicer JD, Yost CC, Weber A, Zuo Y, Egeblad M. Targeting potential drivers of COVID-19: neutrophil extracellular traps. J Exp Med. 2020;217:e20200652.

78. Mauvais-Jarvis F. Aging, male sex, obesity, and metabolic inflammation create the perfect storm for COVID-19. Diab. 2020;69:1857-63.

79. Soares FH, de Sousa MB. Different types of physical activity on inflammatory biomarkers in women with or without metabolic disorders: a systematic review. Women Hlth. 2013;53:298-316.

80. Agarwal D, Haque M, Sriramula S, Mariappan N, Pariaut R, Francis J. Role of proinflammatory cytokines and redox homeostasis in exercise-induced delayed progression of hypertension in spontaneously hypertensive rats. Hypertens. 2009;54:1393-400.

81. Chae CU, Lee RT, Rifai N, Ridker PM. Blood pressure and inflammation in apparently healthy men. Hypertens. 2001;38:399-403.

82. Sabidó M, Hohenberger T, Grassi G. Pharmacological intervention in hypertension using beta-blockers: real-world evidence for long-term effectiveness. Pharmacol Res. 2018;130:191-7.

83. Silva IVG, de Figueiredo RC, Rios DRA. Effect of different classes of antihypertensive drugs on endothelial function and inflammation. Int J Mol Sci. 2019;20:E3458.

84. Cozza IC, Di Sacco TH, Mazon JH, Salgado MC, Dutra SG, Cesarino EJ, Souza HC. Physical exercise improves cardiac autonomic modulation in hypertensive patients independently of angiotensin-converting enzyme inhibitor treatment. Hypertens Res. 2012;35:82-7.

85. Fleshner M. Exercise and neuroendocrine regulation of antibody production: protective effect of physical activity on stress-induced suppression of the specific antibody response. Int J Sports Med. 2000;21:14-9.

86. MacDonald JR, MacDougall JD, Interisano SA, Smith KM, McCartney N, Moroz JS, Younglai EV, Tarnopolsky MA. Hypotension following mild bouts of resistance exercise and submaximal dynamic exercise. Eur J Appl Physiol. 1999;79:148-54.

87. Hjelstuen A, Anderssen SA, Holme I, Seljeflot I, Klemsdal TO. Markers of inflammation are inversely related to physical activity and fitness in sedentary men with treated hypertension. Am J Hypertens. 2006;19:669-75.

88. Donges CE, Duffield R. Effects of resistance or aerobic exercise training on total and regional body composition in sedentary overweight middle-aged adults. Appl Physiol Nut Metabol. 2012;37:499-509.

89. Donges CE, Duffield R, Drinkwater EJ. Effects of resistance or aerobic exercise training on interleukin-6, C-reactive protein, and body composition. Med Sci Sports Exerc. 2010;42:304-13.

90. Coppack SW. Pro-inflammatory cytokines and adipose tissue. Proc Nut Soc. 2001;60:349-56.

91. Mendham AE, Duffield R, Marino F, Coutts AJ. Small-sided games training reduces CRP, IL-6 and leptin in sedentary, middleaged men. Eur J Appl Physiol. 2014;114:2289-97.

92. Mendham AE, Duffield R, Marino F, Coutts AJ. Differences in post-exercise inflammatory and glucose regulatory response between sedentary indigenous australian and caucasian men completing a single bout of cycling. Am J Hum Biol. 2014;26:208-14.

93. Pan D, Sze S, Minhas JS, Bangash MN, Pareek N, Divall P, Williams CML, Oggioni MR, Squire IB, Nellums LB. The impact of ethnicity on clinical outcomes in COVID-19: a systematic review. EClinicalMedicine. 2020;23:100404.

94. Yehia BR, Winegar A, Fogel R, Fakih M, Ottenbacher A, Jesser C, Bufalino A, Huang RH, Cacchione J. Association of race with mortality among patients hospitalized with coronavirus disease 2019 (COVID-19) at 92 US Hospitals. JAMA Net Open. 2020;3:e2018039.

Publisher's Note Springer Nature remains neutral with regard to jurisdictional claims in published maps and institutional affiliations. 